\title{
Association of $\mathrm{THO} 1$ with human longevity revisited
}

\author{
Nicole von Wurmb-Schwark ${ }^{1}$, Amke Caliebe ${ }^{2}$, Thorsten Schwark ${ }^{1}$, Rabea Kleindorp ${ }^{3}$, Micaela Poetsch ${ }^{4}$, \\ Stefan Schreiber ${ }^{3,5}$ and Almut Nebel ${ }^{*, 3}$
}

The gene tyrosine hydroxylase 1 ( $T H O 1$ ) has been suggested as a candidate for human longevity. A previous study has shown an association between longevity and specific alleles of the THO1 short tandem repeat (STR) polymorphism in an Italian population. This STR locus is also widely used in forensic genetics. If the THO1-longevity association could be confirmed in independent samples, this finding would have important ramifications for the use of this polymorphism in a forensic context. In the present study, we sought to replicate the previous association result by investigating 471 long-lived individuals (96-110 years) and 462 younger controls (19-75 years) from Germany. In the analyzed samples, the association between THO1 and longevity was not replicated. However, the obtained THO1 allele frequencies were consistent with published data. We observed considerable differences in the allele distribution between Germans and Italians, in particular with regard to allele 9.3, which displayed a previously undetected decreasing West-East and North-South cline across Europe. The discrepant TH01-longevity association results in Germans and Italians could therefore be due to population-specific effects. This finding highlights the need to take into consideration population genetic data when dealing with association studies.

European Journal of Human Genetics (2011) 19, 924-927; doi:10.1038/ejhg.2011.43; published online 16 March 2011

Keywords: THO1; human longevity; forensic; STR; allele 9.3

\section{INTRODUCTION}

The gene tyrosine hydroxylase 1 (TH01) has been suggested as a candidate for human longevity. Given its role in stress response, it is conceivable that allelic variation in this gene influences survival into old age. A previous study investigated a possible association of the short tandem repeat (STR) polymorphism in intron 1 of THO1 (localization: 11p15.5-p15, motif: TCAT) with longevity in Italians. ${ }^{1}$ The authors reported a statistically significant reduction in the frequency of homozygotes of long THO1 tetranucleotide repeats $(\geq 9)$ in male centenarians compared with younger men. There was no association in females. Subsequent THO1-longevity studies, which differed from the original investigation ${ }^{1}$ in terms of study design, the analysis approaches and examined markers, described contradictory findings. ${ }^{2-4}$ For instance, Tan $e^{2} \mathrm{al}^{3}$ observed an increase in carriers of the long allele 9.3 in Italian centenarians versus younger individuals. In summary, the involvement of THO1 variation in human longevity still remains unresolved. However, if this association could be confirmed, this would have important ramifications for the use of this locus in forensic genetics.

The TH01-STR is one of the most widely used markers in forensic casework. It is a mandatory locus in many forensic databases (eg, CODIS in the US, DAD in Germany, UK Forensic Database in Great Britain) and has therefore been included in most commercially available STR multiplex PCR kits. ${ }^{5}$ However, in recent years, THO1 has not only been linked to human longevity but was also described to be associated with psychiatric disorders ${ }^{6}$ and sudden infant death syndrome. ${ }^{7}$ Other studies investigated a possible role of THO1 in arterial hypertension ${ }^{8,9}$ or smoking, ${ }^{10-13}$ albeit with conflicting results. As a forensically employed genetic polymorphism - at least in Germany - must not reveal information about phenotype, personality or disease susceptibility of the tested individual, ${ }^{14}$ a confirmed association between THO1 alleles and longevity could result in the exclusion of this marker from forensic investigations.

In view of the important role of the THO1 locus in legal medicine and the implications a genuine association would have, we sought to replicate, as primary end point, the initial finding in Italians ${ }^{1}$ in our collection of 471 long-lived individuals (LLI) and 462 younger controls from Germany. Furthermore, we investigated whether allele 9.3 exerts a role as a modulator of longevity as suggested by Tan et al. ${ }^{3}$

\section{MATERIALS AND METHODS}

Study population

Four hundred seventy-one unrelated German LLI between 96 and 110 years of age at recruitment (median age: 101 years) were studied. Three hundred fiftyfour $(75 \%)$ of the LLI were centenarians. The sample of younger German controls was obtained from the popgen biobank ${ }^{15}$ and comprised 462 living subjects aged between 19 and 75 years (median age: 59 years). The controls were matched to the LLI in terms of ancestry and gender, thus minimizing any systematic genetic differences between these samples that might arise due to very low levels of undetected population structure. Based on current predictions only $1.5 \%$ of all 60 -year-old and $1.8 \%$ of all 75 -year-old German females will live up to 100 years. For males, the probability is even lower (Human Mortality Database; http://www.demogr.mpg.de). Thus, fewer than eight of the younger controls are estimated to become centenarians themselves, a negligible proportion that does not affect power. The recruitment procedures for LLI and

\footnotetext{
${ }^{1}$ Institute of Legal Medicine, Christian-Albrechts-University and the University Hospital Schleswig-Holstein, Kiel, Germany; ${ }^{2}$ Institute of Medical Informatics and Statistics, Christian-Albrechts-University and the University Hospital Schleswig-Holstein, Kiel, Germany; ${ }^{3}$ Institute of Clinical Molecular Biology, Christian-Albrechts-University and the University Hospital Schleswig-Holstein, Kiel, Germany; ${ }^{4}$ Institute of Legal Medicine, University Hospital Essen, Essen, Germany; ${ }^{5}$ Popgen Biobank, Christian-AlbrechtsUniversity and the University Hospital Schleswig-Holstein, Kiel, Germany

*Correspondence: Dr A Nebel, Institute of Clinical Molecular Biology, Christian-Albrechts-University, Schittenhelmstrasse 12,24105 Kiel, Germany. Tel: +49 431 597 1373 ; Fax: +49 431597 2196; E-mail: a.nebel@mucosa.de

Received 17 September 2010; revised 20 January 2011; accepted 9 February 2011; published online 16 March 2011
} 
controls have been described in detail elsewhere. ${ }^{16,17}$ Most of the participants provided, as part of a health and family history questionnaire, information about their nicotine consumption habits and arterial hypertension status. The study was approved by the Ethics Committee of the University Hospital Schleswig-Holstein in Kiel.

\section{TH01 typing}

Five nanograms of genomic DNA from each individual, extracted from wholeblood samples, was subjected to a duplex PCR amplifying a TH01-specific STR fragment and the gender-determining amelogenin locus. Primers and PCR conditions were used as described previously. ${ }^{18}$ Amplification products were separated and detected on an ABIPrism3130 Genetic Analyzer with ROX500 standard fragments (all Applied Biosystems, Weiterstadt, Germany) in comparison with a self-made allelic ladder. Electrophoresis results were analyzed using the GeneMapper ID Software v3.2 with the respective self-designed bin set. Allele peaks were scored when the relative fluorescence units were between 50 and 3000 .

\section{Statistical analysis}

The primary aim of the study was to try to validate the result of De Benedictis et al. ${ }^{1}$ Secondary end points of the study were other potential differences in the TH01 allele distribution between cases and controls, with a special focus on allele 9.3. All $P$ values were two-sided and were considered significant if smaller than 0.05 . For the primary end point, only one test was performed. Owing to the exploratory nature of the study with respect to the secondary end points, no adjustment for multiple testing was conducted.

Alleles were grouped according to the previous study of De Benedictis et al: ${ }^{1}$ small (S) - 5, 6, 7, 8; large (L) - 9, 9.3, 10. The alleles ' $S$ ' and 'L' are designated in the following as grouped alleles. The allele ' $10-1$ ' of De Benedictis et al, ${ }^{1}$ also called ' $10^{*}$ ' by Tan $e t a l,{ }^{3}$ is referred to as ' $9.3^{\prime}$ ' in the forensic literature ${ }^{19}$ and in this study. Allele and genotype distributions between cases and controls were compared by applying Fisher's exact test. Independence of the occurrence of allele 9.3 and other alleles in the same genotype was tested with a $\chi^{2}$ goodness-of-fit test. This coincided with a test for Hardy-Weinberg equilibrium. Logistic regression was performed with a carrier status of allele 9.3 as outcome and age as continuous influence variable. The distribution of 9.3 carriers was also studied in four different age groups. Sample size and power calculations were done with BIAS for Windows 8.03. For all other statistical calculations the statistics program $\mathrm{R}$ was used (version 2.5.1 ${ }^{20}$ ). For tests for Hardy-Weinberg equilibrium the package 'genetics' was applied: HWE.exact for two alleles and HWE.chisq for more than two alleles (R package version 1.3.0, 2007).

Smoking and high blood pressure both affect mortality and ageing and may be associated with variation in the THO1 gene. ${ }^{7,12,13}$ To investigate the robustness of our results and to prevent confounding, we repeated the analysis considering only non-smokers $(n=470)$ and individuals free of hypertension $(n=550)$.

\section{RESULTS}

In the German samples, seven alleles were observed at the THO1 locus in both LLI and controls (Figure 1). No significant deviation from Hardy-Weinberg equilibrium was found in either group $(P>0.05)$.

For our primary end point, we applied the same study design as described in De Benedictis et $a l^{1}$ and grouped the alleles as small (S) or large (L). In De Benedictis et al ${ }^{1}$ it was shown that the LL genotype was significantly less frequent in male centenarians when compared with the other two genotypes, SL and SS. For a significance level of 0.05 , our samples had a power of over 99 or $89 \%$, respectively, to replicate the previous results observed for South Italian males and females. ${ }^{1}$ Despite sufficient power, the association between THO1 and longevity was not replicated in the studied German samples ( $P$ values for comparison of LL versus SL/SS: 0.25 for males, 0.91 for females; Table 1). Furthermore, we repeated our analysis for the subgroup of centenarian male cases (the German female cases were all centenarians). The result was again non-significant (relative LL frequency in

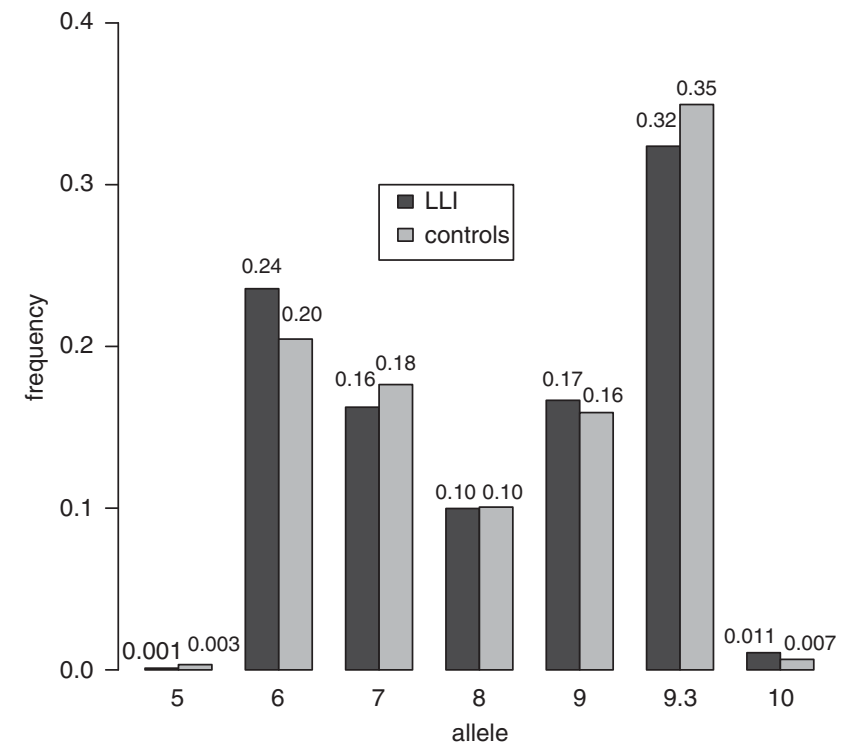

Figure 1 Relative frequencies of ungrouped alleles of the THO1 STR in German LLI and younger controls.

LLI: 0.30, in controls: $0.21, P$-value for comparison: 0.067$)$. The frequency of the LL genotype was higher in German LLI than in controls, in contrast to the result in Italians. ${ }^{1}$

In our sample, the distribution of the ungrouped allele and genotype frequencies in cases and younger controls was very similar (Figure 1). An overall comparison of allele or genotype frequencies showed no significant difference between the two groups (alleles: $P=0.48$, genotypes: $P=0.71$ ).

Allele 9.3 was the most common one in Germans (Figure 1). Tan et $a l^{3}$ reported an increase in carriers of allele 9.3 in Italian centenarians versus younger controls. When 9.3 was analyzed separately against all other alleles in the German sample, no significant deviation between cases and controls was found $(P=0.24)$. In addition, there was no significant result of the increased presence of 9.3 with any of the other alleles in a heterozygote genotype $(P>0.05$ for all other alleles). Furthermore, logistic regression analysis, in which the 9.3 carrier status was considered as outcome and age as continuous influence variable, showed no significant result $(P=0.73)$. Also, the frequency distribution of 9.3 carriers in the four examined age groups was very similar and appeared to fluctuate randomly $(P=0.16$; Table 2). The frequency of carriers in the centenarian group was lower than in the younger age groups, in contrast to previous findings. ${ }^{3}$

When the data were analyzed excluding smokers and individuals with high blood pressure, the results for the primary and secondary end points of the study were similar to those obtained considering all individuals. The results for the primary end point are shown in Table 3 and for detailed subgroup analyses in the Supplementary Material.

The THO1 allele distribution differed considerably between Germans and Italians (Table 4), with the former exhibiting higher frequencies of alleles 9.3 and 10 while in the latter allele 6 is more common. However, our dataset is consistent with that obtained from a large representative sample from Germany and the allele distribution described by Tan $e t a^{3}$ is in accordance with other data derived from Italy (Table 4). These findings suggest population-specific differences and ascertain the accuracy of the obtained data in both our and the Italian study. ${ }^{3}$ 
Table 1 Genotype distribution of grouped THO1 STR alleles in German long-lived individuals and younger controls

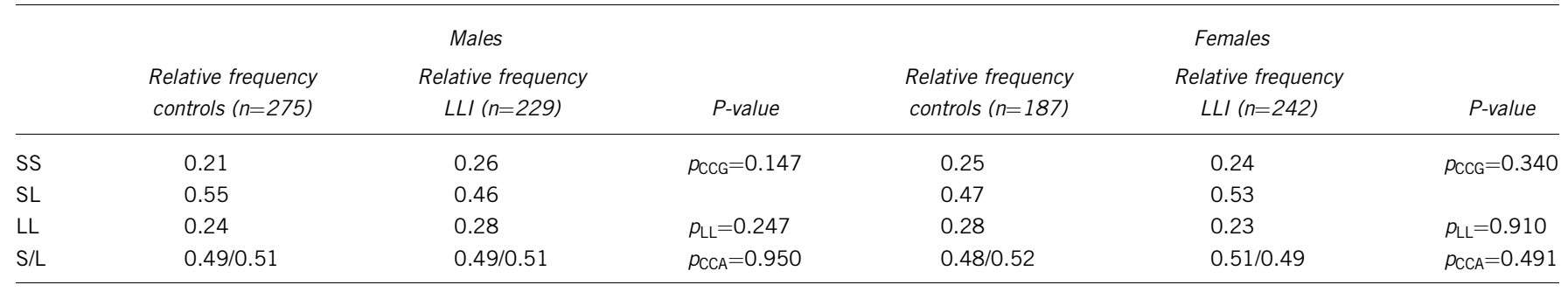

Abbreviations: $n$, number of individuals; LLI, long-lived individuals; S, small allele $(5,6,7,8)$; L, long allele $(9,9.3,10)$; $p_{\text {CCG }}, P$-value for overall genotype frequency comparison of controls and $\mathrm{LLI} ; p_{\mathrm{LL}}, P$-value for frequency comparison of genotype LL against the other two genotypes SL and SS of controls and LLI; $p_{\mathrm{CCA}}, P$-value for allele frequency comparison of controls and LLI.

Table 2 Frequency distribution of carriers of the THO1 STR allele 9.3 in four age groups

\begin{tabular}{lcc}
\hline & \multicolumn{2}{c}{ Carriers of allele 9.3 } \\
Age groups in years $(n)$ & Absolute frequency & Relative frequency \\
\hline $19-59(234)$ & 126 & 0.54 \\
$60-75(228)$ & 141 & 0.62 \\
$96-99(117)$ & 70 & 0.60 \\
$\geq 100(354)$ & 189 & 0.53 \\
\hline
\end{tabular}

Abbreviation: $n$, number of individuals.

Table 3 Results for comparison of grouped THO1 allele frequencies in subsamples of LLI and controls

\begin{tabular}{|c|c|c|c|c|c|c|}
\hline \multirow[b]{2}{*}{ Analyzed subsamples } & \multicolumn{2}{|c|}{$p_{C C A}$} & \multicolumn{2}{|c|}{$p_{C C G}$} & \multicolumn{2}{|c|}{$p_{L L}$} \\
\hline & Males & Females & Males & Females & Males & Females \\
\hline All individuals & 0.949 & 0.491 & 0.147 & 0.340 & 0.250 & 0.910 \\
\hline Non-smokers & 0.831 & 0.432 & 0.224 & 0.553 & 0.237 & 0.876 \\
\hline $\begin{array}{l}\text { Individuals free of } \\
\text { hypertension }\end{array}$ & 0.618 & 0.792 & 0.810 & 0.750 & 0.890 & 0.561 \\
\hline $\begin{array}{l}\text { Non-smokers free of } \\
\text { hypertension }\end{array}$ & 1.000 & 0.913 & 1.000 & 0.916 & 1.000 & 1.000 \\
\hline
\end{tabular}

For abbreviations, see Table 1.

Table 4 Frequency distribution of THO1 alleles in Germans and Italians

\begin{tabular}{llccc}
\hline \multicolumn{2}{c}{$\begin{array}{c}\text { Germans }(\mathrm{n}=933), \text { this } \\
\text { Allele }\end{array}$} & $\begin{array}{c}\text { Germans } \\
(n=10285)^{22}\end{array}$ & $\begin{array}{c}\text { Italians } \\
(\mathrm{n}=965)^{\mathrm{a}}\end{array}$ & $\begin{array}{c}\text { Italians } \\
(\mathrm{n}=6045)^{22}\end{array}$ \\
\hline 4 & 0.00 & 0.00 & 0.00 & 0.0001 \\
5 & 0.0021 & 0.036 & 0.00 & 0.0012 \\
6 & 0.22 & 0.22 & 0.29 & 0.26 \\
7 & 0.17 & 0.16 & 0.16 & 0.16 \\
8 & 0.10 & 0.12 & 0.12 & 0.13 \\
9 & 0.16 & 0.16 & 0.20 & 0.19 \\
9.3 & 0.34 & $0.33^{\mathrm{b}}$ & 0.22 & $0.26^{\mathrm{b}}$ \\
10 & 0.0086 & & 0.017 & \\
\hline
\end{tabular}

aData as listed in Table 1 of Tan et al. ${ }^{3}$

bOnly pooled data (allele 9.3 plus allele 10 ) are available.

\section{DISCUSSION}

Data from our samples of centenarians, nonagenarians and younger controls suggest that the THO1 STR locus exhibits no significant influence on the ability of attaining exceptional old age in Germans. In a previous study, $\mathrm{THO}$ was reported to be associated with longevity in Italian men. ${ }^{1}$ A statistically significant decrease in the frequency of long alleles $(\geq 9)$ was observed in centenarians compared with younger males from the northern ( 26 centenarians versus 49 controls) and southern (27 centenarians versus 113 controls) parts of the country. This finding would indicate a potential survival disadvantage for LL carriers. On the other hand, a later study on an extended and updated version of the original De Benedictis' data described an increase in carriers of allele 9.3 in centenarians $(n=210)$ versus younger individuals $(n=755) .^{3}$ This result suggests a beneficial effect of 9.3, a long allele, which seems to contradict the earlier finding. ${ }^{1}$ However, in our collections, there was no evidence for an association between long alleles of TH01, or specifically allele 9.3, and old age (Tables 1 and 2). The first association reported in Italians might reflect a potentially false-positive result caused by the very small sample sizes examined. ${ }^{1}$ The discrepant finding between Italians as reported by Tan et $a l^{3}$ and Germans (our study) could be due to a population-specific effect. Allele 9.3 is much less common in Italians $(22 \%$, Table 4$)$ than in Germans (34\%, Table 4). This remarkable frequency difference is consistent with other published data from the same populations (Table 4). The distribution of allele 9.3 differs remarkably among European populations, displaying a geographic gradient of decreasing frequency from West to East and North to South (Figure 2). If the TH01 STR is selectively neutral, the observed cline could be a consequence of past migrations or random genetic drift. But since the STR may regulate gene transcription in an allele-specific manner ${ }^{21}$ and may therefore be phenotypically relevant, the geographic allele distribution might very well reflect the adaptation to different environments and selective pressures. More detailed functional studies together with population genetic analyses are necessary to clarify this question.

Taken together, our results do not provide evidence for the involvement of the THO1 locus in the modulation of human longevity in Germans. However, this finding does not rule out the intriguing possibility that such an association may exist in southern and/or eastern European populations. Furthermore, it highlights the need to take into consideration population genetic data when dealing with association studies. The lack of the THO1-longevity association in the investigated German sample means that - at least based on our current knowledge - the forensic application of this robust and widely used marker is still legitimate. 


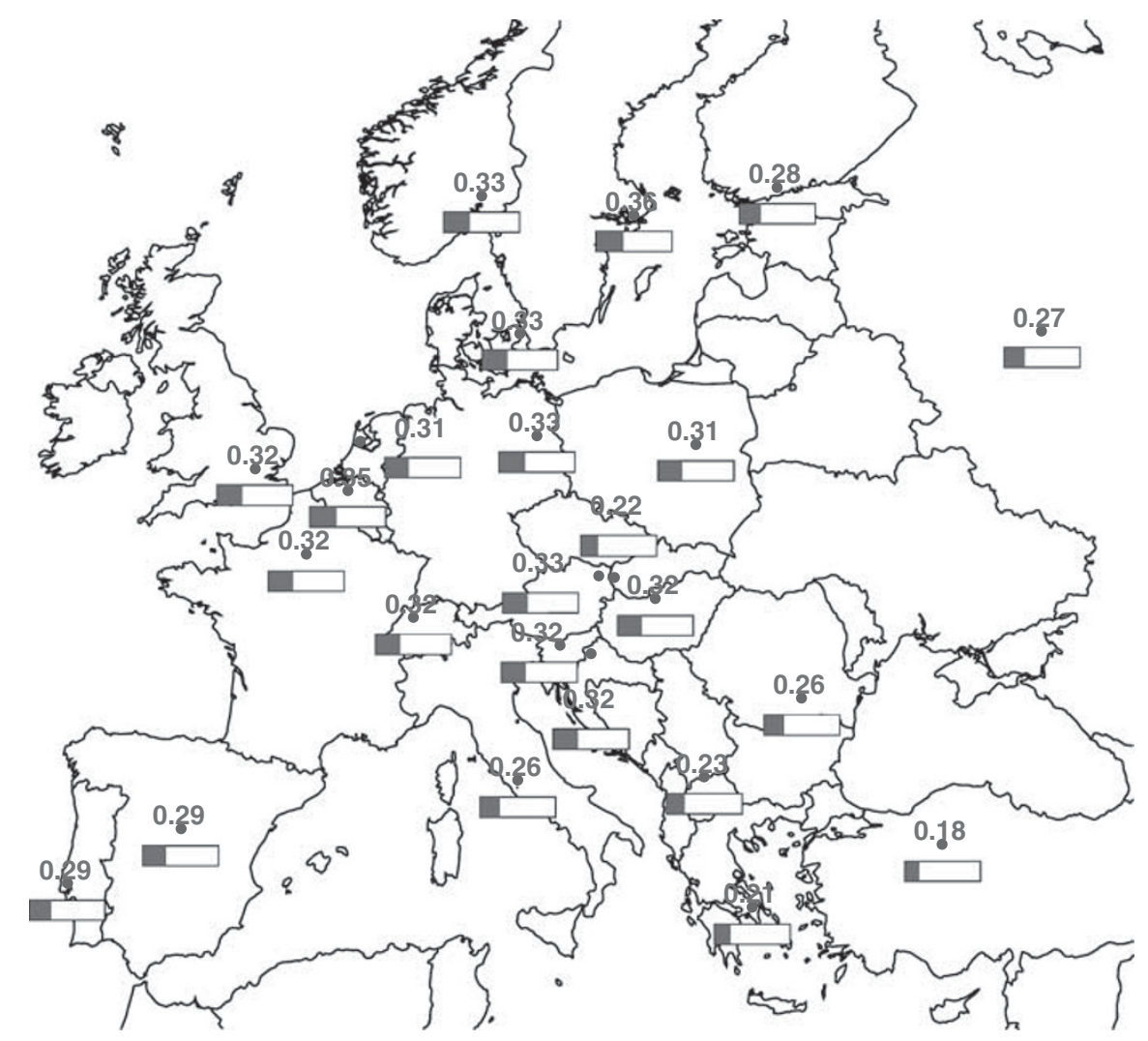

Figure 2 Map showing the frequency distribution of alleles 9.3 and 10 across Europe. For the comparison, allele 9.3 was considered together with the rare allele 10 as for many of the populations studied here no data are available for 9.3 separately. Data are from Huckenbeck et al. ${ }^{22}$

\section{CONFLICT OF INTEREST}

The authors declare no conflict of interest.

\section{ACKNOWLEDGEMENTS}

This study was supported by the DFG Excellence Cluster 'Inflammation at Interfaces'.

1 De Benedictis G, Carotenuto L, Carrieri G et al: Gene/longevity association studies at four autosomal loci (REN, THO, PARP, SOD2). Eur J Hum Genet 1998; 6: 534-541.

2 De Luca M, Rose G, Bonafe M et al: Sex-specific longevity associations defined by tyrosine hydroxylase-insulin-insulin growth factor 2 haplotypes on the $11 \mathrm{p} 15.5$ chromosomal region. Exp Gerontol 2001; 36: 1663-1671.

3 Tan $Q$, Bellizzi D, Rose $G$ et al: The influences on human longevity by HUMTHO1.STR polymorphism (tyrosine hydroxylase gene). A relative risk approach. Mech Ageing Dev 2002; 123: 1403-1410.

4 Lescai F, Blanche H, Nebel A et al: Human longevity and 11p15.5: a study in 1321 centenarians. Eur J Hum Genet 2009; 17: 1515-1519.

5 Gill P, Fereday L, Morling N, Schneider PM: The evolution of DNA databasesrecommendations for new European STR loci. Forensic Sci Int 2006; 156: 242-244.

6 Jacewicz R, Szram S, Galecki P, Berent J: Will genetic polymorphism of tetranucleotide sequences help in the diagnostics of major psychiatric disorders? Forensic Sci Int 2006; 162: 24-27.

7 Klintschar M, Reichenpfader B, Saternus KS: A functional polymorphism in the tyrosine hydroxylase gene indicates a role of noradrenalinergic signaling in sudden infant death syndrome. J Pediatr 2008; 153: 190-193.

8 Sharma $\mathrm{P}$, Hingorani $\mathrm{A}$, Jia $\mathrm{H}$ et al: Positive association of tyrosine hydroxylase microsatellite marker to essential hypertension. Hypertension 1998; 32: 676-682.

9 Klintschar M, Immel UD, Stiller D, Kleiber M: THO1, a tetrameric short tandem repeat locus in the tyrosine hydroxylase gene: association with myocardial hypertrophy and death from myocardial infarction? Dis Markers 2005; 21: 9-13.
10 Lerman C, Shields PG, Main D et al: Lack of association of tyrosine hydroxylase genetic polymorphism with cigarette smoking. Pharmacogenetics 1997; 7: 521-524.

11 Olsson C, Anney R, Forrest S et al: Association between dependent smoking and a polymorphism in the tyrosine hydroxylase gene in a prospective population-based study of adolescent health. Behav Genet 2004; 34: 85-91.

12 Anney RJ, Olsson CA, Lotfi-Miri M, Patton GC, Williamson R: Nicotine dependence in a prospective population-based study of adolescents: the protective role of a functional tyrosine hydroxylase polymorphism. Pharmacogenetics 2004; 14: 73-81.

13 Rodriguez S, Huang S, Chen XH et al: A study of THO1 and IGF2-INS-TH haplotypes in relation to smoking initiation in three independent surveys. Pharmacogenet Genomics 2006; 16: 15-23.

14 Order of the Federal Constitutional Court of Germany (2 BvR 1741/99 9 Dt. Neue Juristische Wochenzeitschr 2000; 12: 882

15 Krawczak M, Nikolaus S, von Eberstein H, Croucher PJ, El Mokhtari NE, Schreiber S: PopGen: population-based recruitment of patients and controls for the analysis of complex genotype-phenotype relationships. Community Genet 2006; 9: 55-61.

16 Nebel A, Croucher PJ, Stiegeler R, Nikolaus S, Krawczak M, Schreiber S: No association between microsomal triglyceride transfer protein (MTP) haplotype and longevity in humans. Proc Natl Acad Sci USA 2005; 102: 7906-7909.

17 Schaefer AS, Richter GM, Nothnagel M et al: A genome-wide association study identifies GLT6D1 as a susceptibility locus for periodontitis. Hum Mol Genet 2010; 19: 553-562.

18 von Wurmb-Schwark N, Preusse-Prange A, Heinrich A, Simeoni E, Bosch T, Schwark T: A new multiplex-PCR comprising autosomal and Y-specific STRs and mitochondrial DNA to analyze highly degraded material. Forensic Sci Int Genet 2009; 3: 96-103.

19 Brinkmann B, Sajantila A, Goedde HW, Matsumoto H, Nishi K, Wiegand P: Population genetic comparisons among eight populations using allele frequency and sequence data from three microsatellite loci. Eur J Hum Genet 1996; 4: 175-182.

20 Team RDC: A Language and Environment for Statistical Computing. R Foundation for Statistical Computing: Vienna, Austria. ISBN: 3-900051-07-0, 2008.

21 Albanese V, Biguet NF, Kiefer H, Bayard E, Mallet J, Meloni R: Quantitative effects on gene silencing by allelic variation at a tetranucleotide microsatellite. Hum Mol Genet 2001; 10: 1785-1792.

22 Huckenbeck W, K K, Scheil HG: The distribution of the human DNA-PCR polymorphisms - a worldwide database. Supplement Volume I/II/III/IV/V/VI/VII (1998/99/00/01/ 02/03/04).

Supplementary Information accompanies the paper on European Journal of Human Genetics website (http://www.nature.com/ejhg) 